\title{
Fake news and indifference to scientific fact: President Trump's confused tweets on global warming, climate change and weather
}

\author{
David E. Allen ${ }^{1,2,3} \cdot$ Michael McAleer $^{2,4,5,6,7}$ (D)
}

Received: 14 June 2018

(C) Akadémiai Kiadó, Budapest, Hungary 2018

\begin{abstract}
A set of 115 tweets on climate change by President Trump, from 2011 to 2015, are analysed by means of the data mining technique, sentiment analysis. The intention is to explore the contents and sentiments of the messages contained, the degree to which they differ, and their implications about his understanding of climate change. The results suggest a predominantly negative emotion in relation to tweets on climate change, but they appear to lack a clear logical framework, and confuse short term variations in localised weather with long term global average climate change.
\end{abstract}

Keywords Sentiment analysis $\cdot$ Polarity $\cdot$ Climate change $\cdot$ Scientific verification $\cdot$ Weather JEL Classification A1 $\cdot \mathrm{C} 88 \cdot \mathrm{C} 44 \cdot \mathrm{Z} 0$

"To permit ignorance is to empower it. To do nothing as our leaders proclaim absurdities is a crime of complacency".

Edmond Kirsch in Origin by Dan Brown.

The analysis in the paper was undertaken with the $\mathrm{R}$ sentiment package.

Michael McAleer

michael.mcaleer@gmail.com

1 School of Mathematics and Statistics, University of Sydney, Sydney, Australia

2 Department of Finance, Asia University, Taichung, Taiwan

3 School of Business and Law, Edith Cowan University, Perth, WA, Australia

4 Discipline of Business Analytics, University of Sydney Business School, Sydney, Australia

5 Institute of Advanced Studies, Yokohama National University, Yokohama, Japan

6 Econometric Institute, Erasmus School of Economics, Erasmus University Rotterdam, Rotterdam, The Netherlands

7 Department of Economic Analysis and ICAE, Complutense University of Madrid, Madrid, Spain 


\section{Introduction}

A series of 115 tweets by President Trump, on the topics of climate change, global warming and the Paris Accord, are analysed by means of textual analysis using data mining techniques. The tweets date from the beginning of 2011 and conclude in October 2015. The analysis features the use of an $\mathrm{R}$ library package which facilitates sentiment analysis, 'sentiment'. The tweets were taken from an on-line sample available at https://www.vox. com/policy-and-politics/2017/6/1/15726472/trump-tweets-global-warming-paris-climateagreement. This period predates his election to the Presidency.

\section{Research method}

The 'sentiment' package was written by De Vries (2012), is now archived from the current release of R, and can be loaded from 'Github.com'. It is a dictionary-based method which calculates sentiment scores using affinity dictionaries. The program splits strings into words (by default at space), looks up an affinity score for each word, and returns the average, using a scale from +5 to -5 . The authors apply this package because it is more finely grained and categorizes five different sentiment emotions, namely joy, sadness, anger, fear and surprise, and reveals greater information about the emotional tenor of the text or string that is analysed.

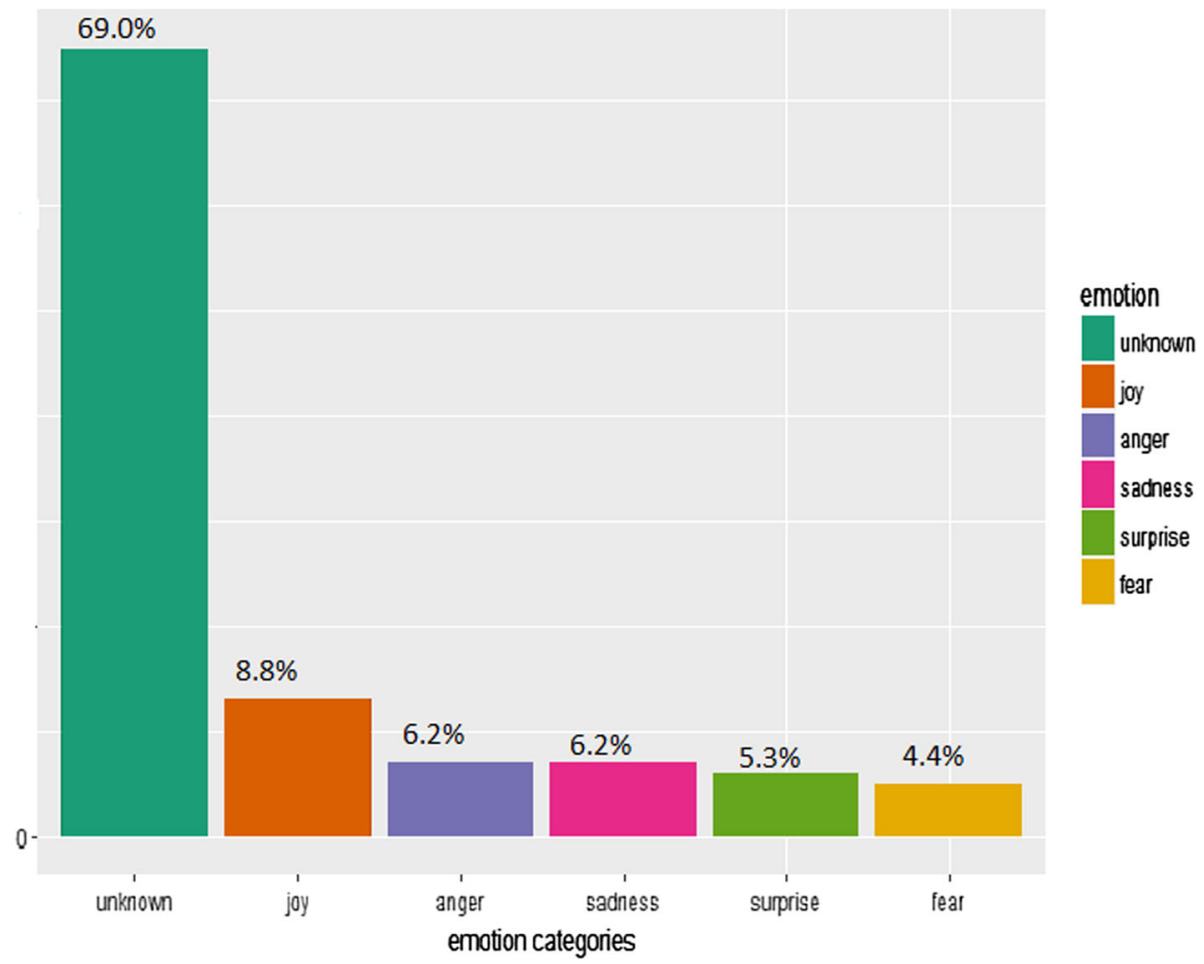

Fig. 1 Trump climate tweet sentiment 


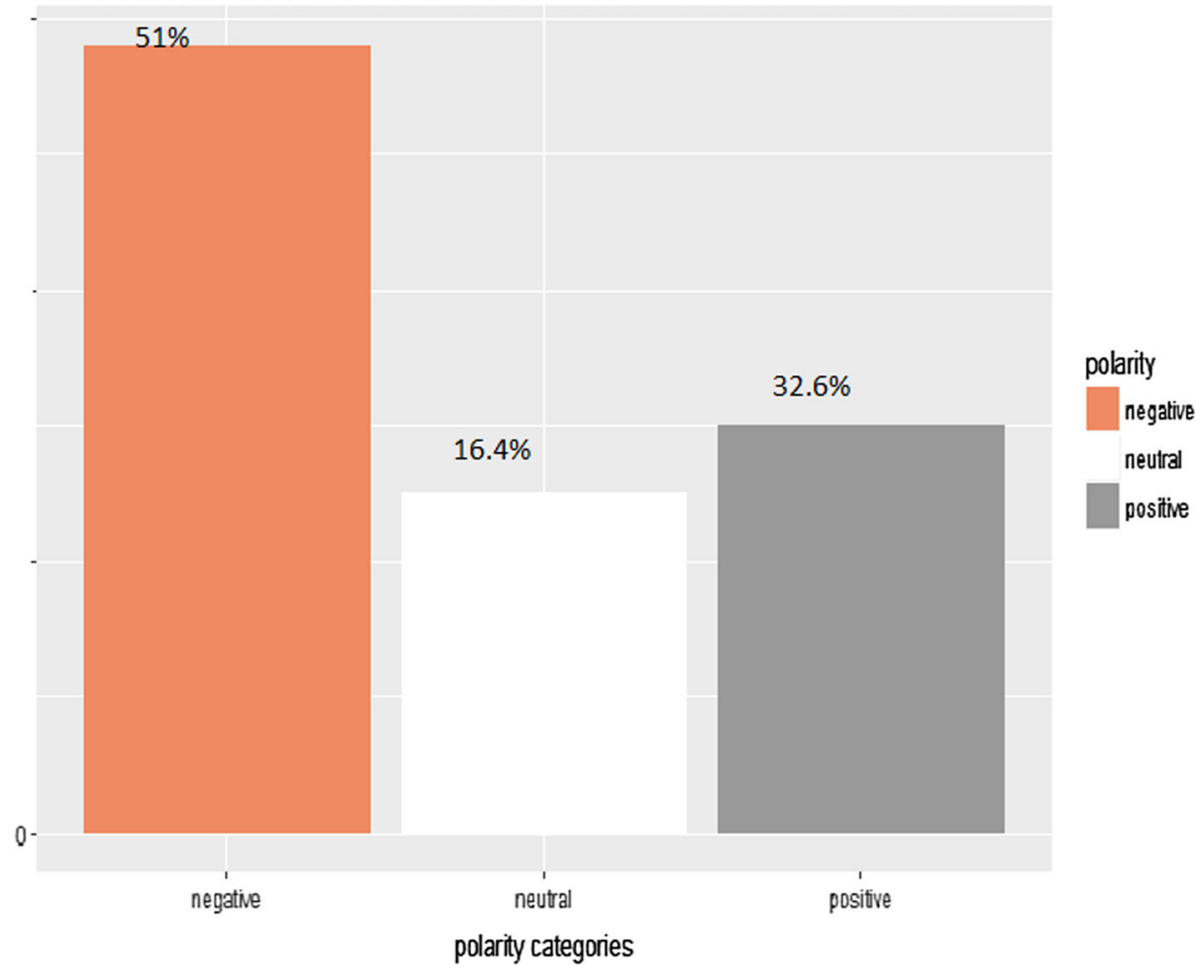

Fig. 2 Trump climate tweet sentiment polarity

The process of performing sentiment analysis requires textual input in a machinereadable format. Pre-processing is required to turn the text into single words, followed by what are common pre-processing steps: stopword removal, stemming, removal of punctuation, and conversion to lower case.

The limitations of the analysis should be borne in mind. The context of 'natural language processing', of which sentiment analysis is a component, is important. The use of sarcasm and other types of ironic language are inherently problematic for machines to detect, when viewed in isolation. This is a potential issue, in particular, in the analysis of President Trump's tweets. Nevertheless, current methods are revealing, as will be seen in the next section which presents the results.

\section{Results of the analysis}

We commence with the results of the application of the sentiment package to President Trump's 115 tweets. The emotional content of these is shown in Fig. 1. Ignoring the 'unknown' category, the predominant emotion recognised in Fig. 1 is 'joy', followed by 'anger', 'sadness', 'surprise', and fear. 69 per cent of the tweets are not classified, but nearly 9 per cent is classified as being 'joy', which is a positive emotion.

Figure 2 classifies the tweets by President Trump according to whether they are negative, neutral or positive. Just over 50 per cent of the classifications in Fig. 2 are negative while around 32 per cent are positive 


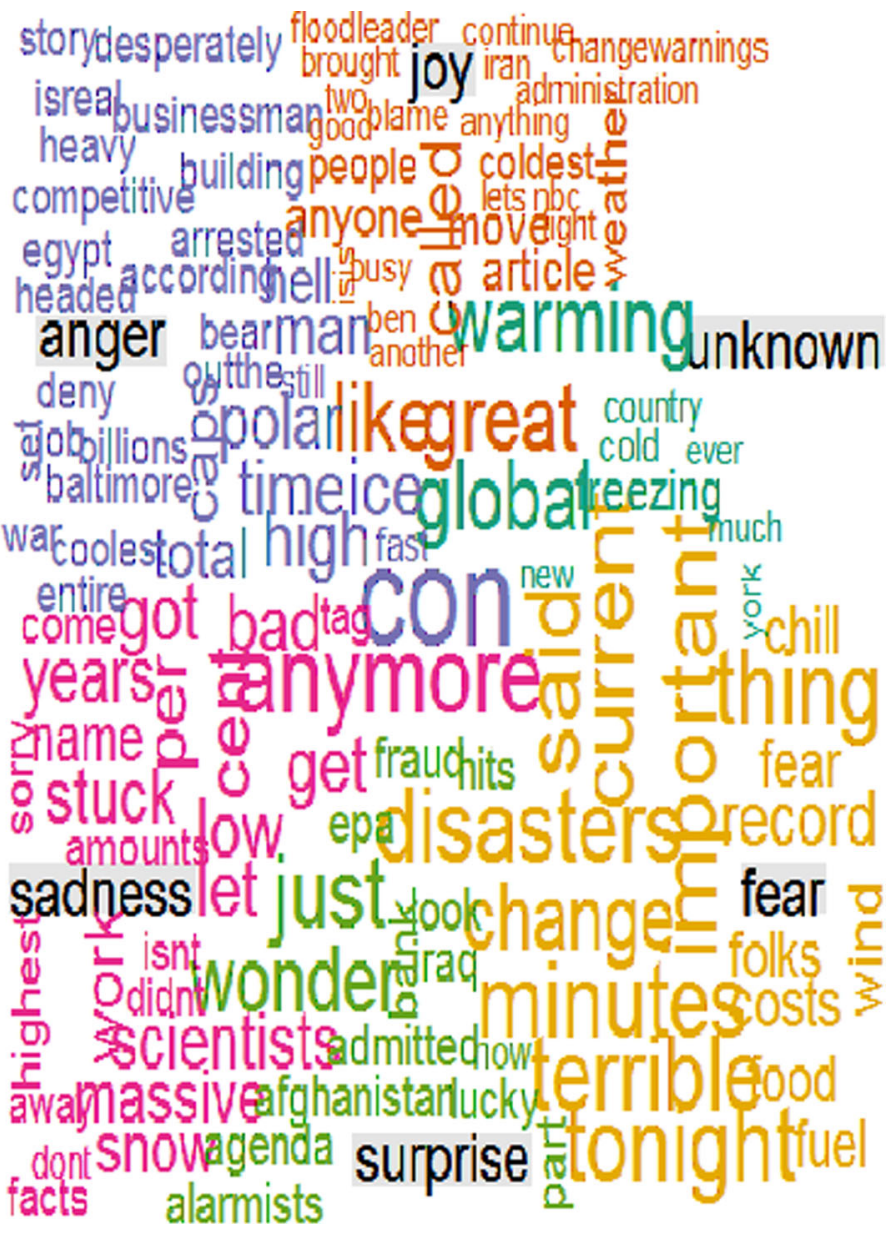

Fig. 3 Trump climate tweets word cloud

Figure 3 shows a word cloud analysis of Trump's tweets. A word cloud is another form of visual representation of text data in which tags are single words, and their relative sizes and colours represent their weighting or importance in the context of the text considered.

The most prominent words in the word cloud in Fig. 3 are 'con', 'global warming' and 'anymore'. If we move around the cloud in an anti-clockwise manner, words in the 'joy' section include 'change warnings', 'coldest', 'weather, 'like', 'great', 'administration', 'called', and so forth. In the 'anger' section below, we have 'story', 'desparately', 'deny', 'billions', 'polar', 'coolest', and so on. In the sadness section, we see 'anymore', 'stuck', 'massive', 'snow', 'low', 'facts', and so on. In the 'surprise' section, we see 'wonder', 'fraud', 'agenda', 'alarmists', 'epa', and so forth. In the 'fear' section, we see 'disaster', 'change', 'minutes', 'record', 'costs', and so on. The 'unknown section' has a diverse grouping of words, with 'global warming' and 'freezing' given noticeable prominence. 


\section{Conclusion}

The results suggest that there is no systematic logical pattern because Trump is confused about weather, Global Warming (which is purportedly "inconsistent with cold weather" !), and Climate Change, which he states incorrectly as another name for Global Warming. In short, there is no logical pattern to his tweets on these topics. This is scientific support for his indifference to scientific fact. Global warming is the observed century-scale rise in the average temperature of the Earth's climate system and its related effects.

The time frame is crucial, namely a "century-scale" time frequency. Climate change is a change in the statistical distribution of weather patterns when that change lasts for an extended period of time, so it refers to a change in average weather conditions over an extended time frame. Trump confuses changes in average weather conditions over an extended time frame with specific daily or hourly weather reports, such as "Breaking News", which is a very short time frequency for individual observations.

It is well known that standard errors are much higher for individual observations based on specific time frequencies as compared with averages over an extended time frequency. This is why "Global Warming" can include extremes in hot and cold weather observations, that is, positive and negative observations in the statistical weather pattern distribution, which Trump always uses as "arguments" against Global Warming. Such extremes are entirely consistent with large standard errors for individual observations, such as daily or hourly weather reports.

Trump's total confusion and complete misunderstanding of the meaning of Global Warming seems to be the sole reason for having withdrawn the USA from the Paris Accord. Very sad!

Acknowledgements For financial support, the first author acknowledges the Australian Research Council, and the second author is most grateful to the Australian Research Council, National Science Council, Ministry of Science and Technology (MOST), Taiwan, and the Japan Society for the Promotion of Science.

\section{References}

De Vries, A. (2012). Sentiment Package, available at https://github.com/andrie/sentiment/blob/master/ DESCRIPTION. 